\title{
Disentangling Orbital and Valley Hall Effects in Bilayers of Transition Metal Dichalcogenides
}

\author{
Tarik P. Cysne $\odot,{ }^{1, *}$ Marcio Costa, ${ }^{1}$ Luis M. Canonico $\odot,{ }^{2}$ M. Buongiorno Nardelli, ${ }^{3}$ \\ R. B. Muniz, ${ }^{1}$ and Tatiana G. Rappoport $\oplus^{4,5}$ \\ ${ }^{1}$ Instituto de Física, Universidade Federal Fluminense, 24210-346 Niterói Rio de Janeiro, Brazil \\ ${ }^{2}$ Catalan Institute of Nanoscience and Nanotechnology (ICN2), CSIC and BIST, Campus UAB, Bellaterra, 08193 Barcelona, Spain \\ ${ }^{3}$ Department of Physics and Department of Chemistry, University of North Texas, Denton, Texas 76203, USA \\ ${ }^{4}$ Instituto de Telecomunicações, Instituto Superior Tecnico, University of Lisbon, Avenida Rovisco Pais 1, Lisboa 1049001, Portugal \\ ${ }^{5}$ Instituto de Física, Universidade Federal do Rio de Janeiro, C.P. 68528, 21941-972 Rio de Janeiro RJ, Brazil
}

(Received 20 October 2020; accepted 7 January 2021; published 5 February 2021)

\begin{abstract}
It has been recently shown that monolayers of transition metal dichalcogenides (TMDs) in the $2 \mathrm{H}$ structural phase exhibit relatively large orbital Hall conductivity plateaus within their energy band gaps, where their spin Hall conductivities vanish [Canonico et al., Phys. Rev. B 101, 161409 (2020); Bhowal and Satpathy, Phys. Rev. B 102, 035409 (2020)]. However, since the valley Hall effect (VHE) in these systems also generates a transverse flow of orbital angular momentum, it becomes experimentally challenging to distinguish between the two effects in these materials. The VHE requires inversion symmetry breaking to occur, which takes place in the TMD monolayers but not in the bilayers. We show that a bilayer of $2 \mathrm{H}-\mathrm{MoS}_{2}$ is an orbital Hall insulator that exhibits a sizeable orbital Hall effect in the absence of both spin and valley Hall effects. This phase can be characterized by an orbital Chern number that assumes the value $\mathcal{C}_{L}=2$ for the $2 \mathrm{H}-\mathrm{MoS}_{2}$ bilayer and $\mathcal{C}_{L}=1$ for the monolayer, confirming the topological nature of these orbital-Hall insulator systems. Our results are based on density functional theory and low-energy effective model calculations and strongly suggest that bilayers of TMDs are highly suitable platforms for direct observation of the orbital Hall insulating phase in two-dimensional materials. Implications of our findings for attempts to observe the VHE in TMD bilayers are also discussed.
\end{abstract}

DOI: 10.1103/PhysRevLett.126.056601

Introduction.-The orbital Hall effect (OHE) is the orbital analog of the spin Hall effect and consists in the appearance of a transverse current of orbital angular momentum that is induced by a longitudinally applied electric field [1]. Recently, a renewed interest in orbital magnetism and other orbital effects [2-5] gave origin to various theoretical studies on the OHE and related phenomena [6-15]. The possibility of using the OHE to generate orbital torque in magnetic materials $[16,17]$ motivated new experimental works on orbital dynamics in magnetic multilayers $[18,19]$, raising expectations that orbital angular degrees of freedom may eventually be employed to process information in logic and memory devices.

The interrelation between the OHE and the presence of orbital textures in reciprocal space [10] has been established and characterized both theoretically and experimentally in several low-dimensional materials $[5,6,12,13,20,21]$, widening the class of systems that may be utilized for orbitronic applications. More specifically, the occurrence of a relatively large OHE has been predicted in the $2 \mathrm{H}$ structural phase of transition metal dichalcogenide (TMD) monolayers [6,7], where it is associated with the presence of a Dresselhaus-like orbital texture around the valleys [6]. However, it is experimentally challenging to observe just the OHE in 2H-TMD monolayers due to the concurrent presence of the valley Hall effect (VHE) that also contributes to the transport of orbital angular momentum in these systems [22].

It is noteworthy, though, that the VHE manifests only in the absence of inversion symmetry, which naturally happens for the monolayers, but for bilayers, comprising two monolayers rotated by $\pi$ with respect to each other, the inversion symmetry is restored. This substantially affects valley related phenomena [23-25]. For instance, the valley Hall conductivity has opposite signs in each layer, canceling the VHE for the bilayer [26-28], as we shall subsequently discuss. Nevertheless, it is also possible to break inversion symmetry in the bilayers by applying an electric field perpendicular to the layers, by means of which one can control the valley polarization [29] and the VHE intensity [25] with a gate voltage.

Here, we perform calculations of the orbital Hall conductivities for ultrathin films (single layer and bilayer) of $2 \mathrm{H}-\mathrm{MoS}_{2}$ which is representative of this class of systems. We combine density functional theory (DFT) and an effective low-energy model to disentangle the valley and orbital physics of TMD bilayers and explore some of their topological orbital features. 
Implications of our findings regarding interpretations of recent experiments on the electric control of the VHE in $\mathrm{MoS}_{2}$ bilayers [24,25] are also briefly discussed. Our results strongly indicate that bilayers of TMDs constitute a fertile playground for exploring orbital angular momentum current generation in 2D-like systems.

DFT results.-Our DFT calculations [30,31] were performed with the plane-wave-based code QUANTUM ESPRESSO [32]. The exchange and correlation potential is treated within the generalized gradient approximation [33]. The ionic cores were described with fully relativistic projected augmented wave (PAW) potentials [34]. We used a cutoff energy of 63 Ry for the wave functions and a value 10 times larger for the charge density. In order to reproduce the interlayer distance of the $\mathrm{MoS}_{2}$ bilayer, we have used the DFT-D3 [35] method, which describes reasonably well the van der Walls forces in these systems. We have chosen a $10 \times 10 \times 1$ reciprocal space sampling, and to avoid spurious interaction due to periodic boundary conditions, we insert a vacuum spacing of $15 \AA$. We constructed an effective tight-binding Hamiltonian from our DFT calculations using the pseudoatomic orbital projection (PAO) method [36,37]. The PAO method consists of projecting the DFT Kohn-Sham orbitals into the compact subspace spanned by the pseudoatomic orbitals which are naturally built into the PAW potentials. The PAW potentials used for the Mo and $\mathrm{S}$ were constructed with a $s s p d$ and $s p$ basis, respectively.

Once the PAO Hamiltonian is constructed, we can calculate the spin Hall $(\mathrm{SH})$ and orbital Hall $(\mathrm{OH})$ responses to an applied electric field [1,6,8,13,38-40]. Up to linear order on the external field, they are given by

$$
\sigma_{\mathrm{OH}(\mathrm{SH})}^{\eta}=\frac{e}{(2 \pi)^{2}} \sum_{n} \int_{\mathrm{BZ}} d^{2} k f_{n \vec{k}} \Omega_{n, \vec{k}}^{X_{\eta}}
$$

where $\sigma_{\mathrm{OH}(\mathrm{SH})}^{\eta}$ is the orbital Hall (spin Hall) de conductivity with polarization along the $\eta$ direction, and

$$
\Omega_{n, \vec{k}}^{X_{\eta}}=2 \hbar \sum_{m \neq n} \operatorname{Im}\left[\frac{\left\langle\psi_{n, \vec{k}}\left|j_{y, \vec{k}}^{X_{\eta}}\right| \psi_{m, \vec{k}}\right\rangle\left\langle\psi_{m, \vec{k}}\left|v_{x}(\vec{k})\right| \psi_{n, \vec{k}}\right\rangle}{\left(E_{n, \vec{k}}-E_{m, \vec{k}}+i 0^{+}\right)^{2}}\right],
$$

represents the angular-momentum-weighted Berry curvature $[10,40]$. Here, $E_{n, \vec{k}}$ denotes the eigenvalue of the Hamiltonian $H(\vec{k})$ in reciprocal space, and $\left|\psi_{n, \vec{k}}\right\rangle_{\vec{k}}$ is the corresponding eigenvector; $n$ is the band index, $\vec{k}$ is the wave vector. The velocity operators are defined as $v_{x(y)}(\vec{k})=\partial H(\vec{k}) / \partial \hbar k_{x(y)}$, where $x$ and $y$ specify the Cartesian axes, and we assume that the electric field is applied along the $\hat{x}$ direction. The current density operator component along $\hat{y}$ with polarization $\eta$ is defined as $j_{y, \vec{k}}^{X_{\eta}}=\left[X_{\eta} v_{y}(\vec{k})+v_{y}(\vec{k}) X_{\eta}\right] / 2$, where, for the SH conductivity, $X_{\eta}=\hat{s}_{\eta}$ and for the $\mathrm{OH}$ conductivity (OHC), $X_{\eta}=\hat{\ell}_{\eta} ; \hat{s}_{\eta}$ and $\ell_{\eta}$ represent the $\eta$ components of the spin and of the atomic angular momentum operators, respectively. This is implemented in the PAOFLOW code [41] that has been successfully used to study topological materials $[42,43]$ and time dependent spin dynamics [44] among other topics. For our conductivity calculations, we have increased the sampling to $200 \times 200 \times 1 k$ points in the 2D BZ.

Figures 1(a) and 1(b) illustrate results of our calculations for a monolayer and for a bilayer of $2 \mathrm{H}-\mathrm{MoS}_{2}$, respectively. In the left panels of Fig. 1, we compare the band structures obtained from DFT (purple solid lines) and from PAOFLOW (yellow dashed lines). The agreement between the two approaches is excellent. For the monolayer, we obtain a direct energy-band gap of $1.60 \mathrm{eV}$, whereas for the bilayer, we found an indirect gap of $1.28 \mathrm{eV}$, in agreement with previous calculations [45]. The results for the SH conductivity (SHC) (red solid lines) and for the $\mathrm{OHC}$ (blue lines) are shown in the right-hand side panels of Fig. 1. In accordance with our previous results for the monolayer [6], we note in the right panel of Fig. 1(a) that the SHC vanishes
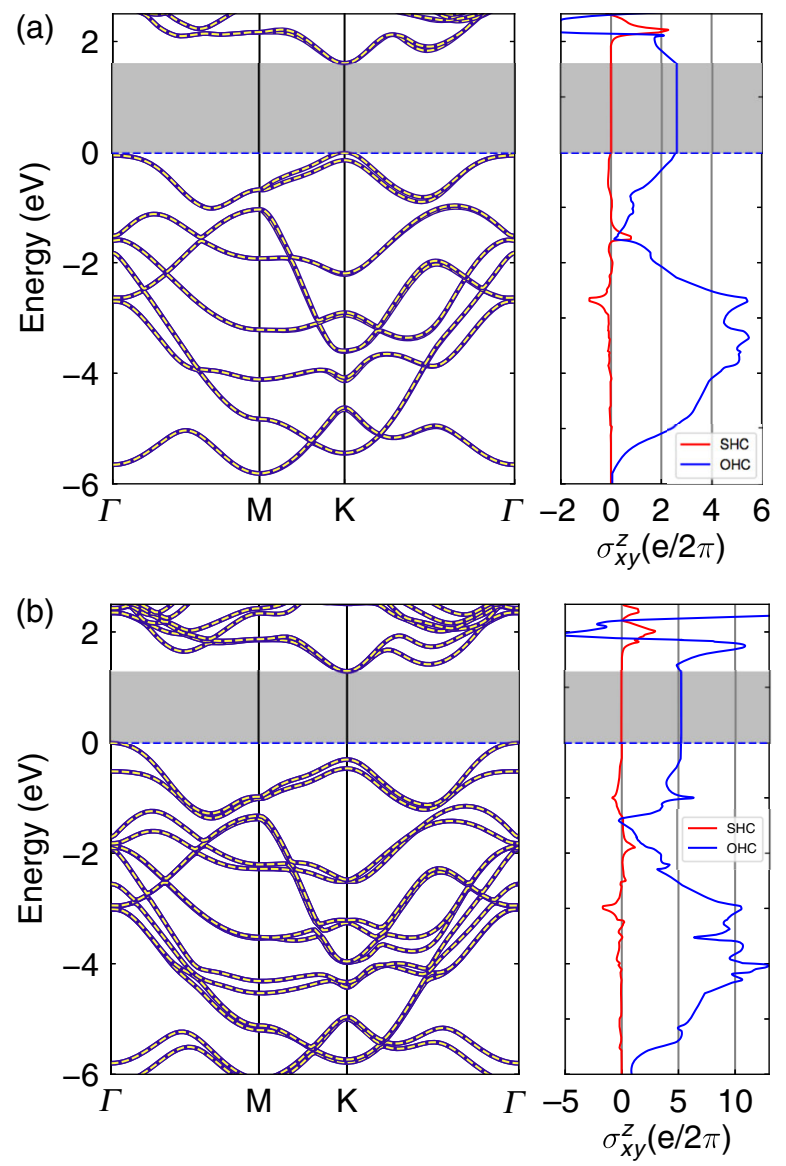

FIG. 1. Energy band structures (left panel) together with the spin-Hall and orbital-Hall conductivities (right panel) calculated for a $\mathrm{MoS}_{2}$ monolayer (a) and for a $\mathrm{MoS}_{2}$ bilayer (b). The purple solid and yellow dashed lines depict the DFT and PAOFLOW band structure calculations, respectively. The horizontal blue dashed line shows the Fermi level. 
in the main energy gap, but the $\mathrm{OHC}$ is finite and exhibits a relatively high plateau of $\approx 2.6(e / 2 \pi)$ in height within this energy range. For the bilayer, however, the right-hand side panel of panel Fig. 1(b) show that the height of the OHC plateau is essentially twice the monolayer value, while the SHC remains null in the main energy gap because it is topologically trivial. Although other regions in the BZ contribute to the $\mathrm{OHC}$ [7], the main contribution originates from the orbitally projected Berry curvature in the vicinity of K and K', as illustrated in the Supplemental Material [46]. To explore the physics behind these results, it is instructive to make use of a low-energy approximation around the $K$ points (valleys) of the BZ, to build a simple model that is able to reasonably describe the main transport characteristics of these systems.

Low energy calculations. - Similar to the monolayers, the low-energy physics of TMD bilayers is dominated by the $d_{z^{2}}, d_{x^{2}-y^{2}}$, and $d_{x y}$ atomic orbitals of the transition metal atoms [26,54,55]. We follow references [26,27] to build a simplified tight-binding (TB) model Hamiltonian in reciprocal space, which is expanded up to first order in the electronic momentum around the valleys located at $\vec{K}=(4 \pi / 3 a) \hat{x}$ and $\vec{K}^{\prime}=-\vec{K}$. This procedure leads to the following Hamiltonian:

$$
\tilde{H}\left(\vec{q}_{\tau}\right)=\left[\begin{array}{cccc}
\Delta & \gamma_{+} & 0 & 0 \\
\gamma_{-} & -\tau s_{z} \lambda & 0 & t_{\perp} \\
0 & 0 & \Delta & \gamma_{-} \\
0 & t_{\perp} & \gamma_{+} & \tau s_{z} \lambda
\end{array}\right],
$$

where $\gamma_{ \pm}=a t\left(\tau q_{x} \pm i q_{y}\right), \tau= \pm 1$ is the valley quantum number associated with valleys $K$ and $K^{\prime}$, respectively. Here, $\vec{k}=\vec{q}+\tau \vec{K}$ where $\vec{q}$ represents the wave vector relative to valleys and $s_{z}$ denotes the usual Pauli matrix. For a $2 \mathrm{H}-\mathrm{MoS}_{2}$ bilayer, an archetypal TMD, $\Delta=1.766 \mathrm{eV}$ is the monolayer band-gap, $a=3.160 \AA$ is the lattice constant, $t=1.137 \mathrm{eV}$ is the intralayer nearest-neighbor hopping, $\lambda=0.073 \mathrm{eV}$ is the spin-orbit coupling, and $t_{\perp}=0.043 \mathrm{eV}$ is the interlayer hopping [26].

The TB basis for this minimal model comprises $\left\{\left|d_{z^{2}}^{1}\right\rangle\right.$, $\left.\left(\left|d_{x^{2}-y^{2}}^{1}\right\rangle-i \tau\left|d_{x y}^{1}\right\rangle\right) / \sqrt{2},\left|d_{z^{2}}^{2}\right\rangle,\left(\left|d_{x^{2}-y^{2}}^{2}\right\rangle+i \tau\left|d_{x y}^{2}\right\rangle\right) / \sqrt{2}\right\}$, where the superscripts 1 and 2 specify the two layers of the bilayer, respectively. It is noteworthy that the orbital angular momentum (OAM) operator in this representation is given by $L_{z}=\operatorname{diag}(0,-2 \hbar \tau, 0,2 \hbar \tau)$, which clearly does not commute with the Hamiltonian defined in Eq. (3).

Equation (2) can be used with the four-band low-energy Hamiltonian given by Eq. (3) to define the Berry and the orbital-weighted Berry curvatures that encode information of the VHE and OHE, respectively. For simplicity, we shall initially neglect the effect of spin-orbit coupling $(\lambda)$, thereby restricting Eq. (3) to a spinless Hamiltonian, and including a degenerescence factor $g_{s}=2$. Equation (2), for
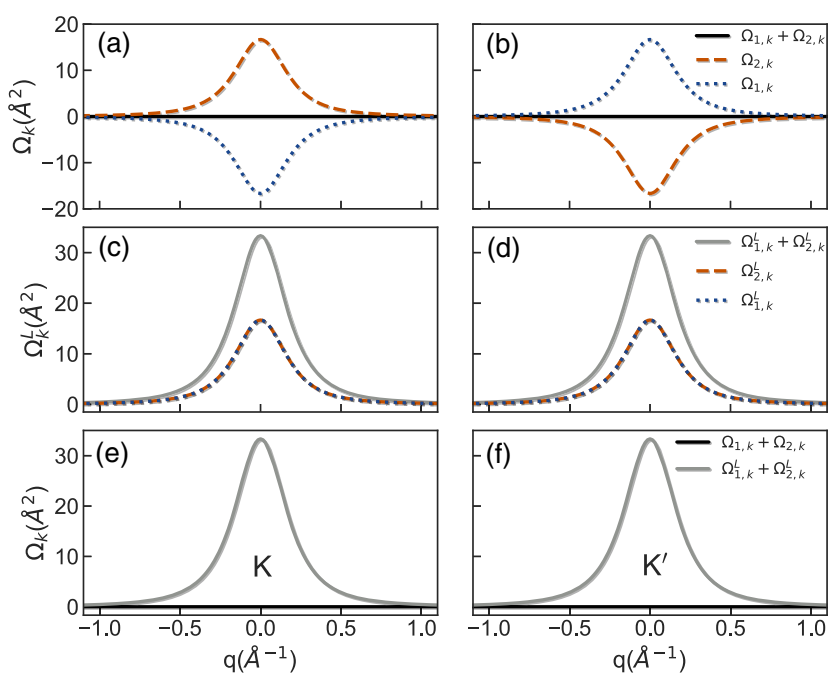

FIG. 2. Berry curvature $\Omega_{n, k}$ at points $K$ (a) and $K^{\prime}$ (b), orbital weighted Berry curvature $\Omega_{n, k}^{L}$ at points $K$ (c) and $K^{\prime}$ (d) for the two valence bands $E_{1}(q)$ and $E_{2}(q)$, associated with the two layers. (e) Total Berry curvature $\Omega_{1, k}+\Omega_{2, k}$ and orbital weighted Berry curvature $\Omega_{1, k}^{L}+\Omega_{2, k}^{L}$ for the bilayer TMD at points $K$ (e) and $K^{\prime}$ (f).

the orbital weighted Berry curvature, may also be employed to calculate the usual Berry curvature $\Omega_{n, k}$, provided that $X_{\eta}$ is replaced by $\hbar \mathbb{1}$. The spinless Hamiltonian generates two valence bands $\left[E_{1}(q)\right.$ and $\left.E_{2}(q)\right]$ that can be regarded as arising from each of the TMD layers because of the relatively small interlayer hopping. Figures 2(a) and 2(b) present the Berry curvatures for both $E_{1}$ and $E_{2}$ calculated around the $K$ and $K^{\prime}$ points, respectively. The Berry curvature for $E_{2}$ has a positive peak at $K$ and a negative peak at $K^{\prime}$, which gives rise to a VHE. The opposite occurs for the Berry curvature of $E_{1}$, which has a negative peak around $K$ and a positive peak at $K^{\prime}$, giving origin to a VHE with an inverted sign. By adding the contributions of both layers, the net Berry curvature is zero in both valleys, and the VHE vanishes. This is a consequence of time-reversal symmetry and the presence of spatial inversion symmetry in the bilayer $[27,54,56]$. A similar situation occurs for TMDs with the $T$ and $T^{\prime}$ structural phases, such as $\mathrm{WTe}_{2}$ [57]. Figures 2(c) and 2(d) show the orbital weighted Berry curvatures for both bands around the $K$ and $K^{\prime}$ points, respectively. In contrast to the previous case, the peaks of the orbital-weighted Berry curvatures for both bands have the same sign around both valleys. Hence, the total orbital-weighted Berry curvature has a finite value, which leads to an $\mathrm{OH}$ insulating phase [6] with no VHE, as Figs. 2(e) and 2(f) illustrate. We note that, in order to assess just the OHE, it is crucial from the experimental point of view to have an OHE without a VHE, because the VHE also leads a transverse angular momentum current $[22,58,59]$ that is hard to distinguish from the one generated by the OHE, as it 
happens for TMD monolayers [6]. Thus, our results show that bilayers of $2 \mathrm{H}$-TMDs are very promising candidates for observing the orbital Hall insulating phase with no interferences from VHE or SH effect.

Now, let us address the topological characterization of the $\mathrm{OH}$ insulating phase in TMD-bilayers. Our Berry curvature analysis suggests that it is possible to associate an orbital Chern number to describe the distinctive nature of these states in analogy with the well known spin Chern number $[60,61]$. Here, the situation is slightly more subtle because the operator $L_{z}$ does not commute with the Hamiltonian of Eq. (3) for finite $\vec{q}$. This is similar to the problem of a quantum spin Hall insulator in the presence of a Rashba spin-orbit coupling. To address this issue, we follow the procedure developed in Refs. [62-64] to define the orbital Chern number $\mathcal{C}_{L}$ for the insulating phase of the Hamiltonian given by Eq. (3). In this formalism, $\mathcal{C}_{L}=$ $\left(\mathcal{C}_{L}^{+}-\mathcal{C}_{L}^{-}\right) / 2$ where $\mathcal{C}_{L}^{ \pm}$are the Chern numbers calculated with the eigenstates of an OAM operator projected on the valence-band states $\left[\mathbb{L}^{\mathrm{v}}(\vec{k})=P(\vec{k}) L_{z} P(\vec{k})\right]$, where $P(\vec{k})$ is the projector operator. If the bands have orbital polarization, the spectrum of $\mathbb{L}^{\mathrm{v}}(\vec{k})$ consists of two groups of eigenvalues $(\epsilon)$ associated with $m_{l}= \pm 2$ that are symmetrically separated by a gap. The projectors on the eigenstates associated with the positive and negative eigenvalues can then be used to calculate the Chern numbers $\mathcal{C}_{L}^{ \pm}$.

Thus, to calculate $\mathcal{C}_{L}$, it is necessary to decompose the valence-band states into two sectors with respect to operator $L_{z}$. For that purpose, first, we obtain the matrix $\mathbb{L}^{\mathrm{v}}(\vec{k})$, with matrix elements given by $\left\langle\psi_{n, \vec{k}}\left|L_{z}\right| \psi_{m, \vec{k}}\right\rangle$, where $n, m$ label the valence-band eigenstates of the low-energy Hamiltonian; more details are given in the accompanying Supplemental Material [46]. It is worth mentioning that, hereafter, we reinstate the spin degree of freedom and the spin-orbit interaction in the Hamiltonian (3). Figure 3(a) shows the eigenvalues of $\mathbb{L}^{\mathrm{v}}(\vec{k})$ calculated as functions of $q$. We clearly see that the spectrum splits in two separated sectors, allowing us to use the eigenstates of $\mathbb{L}^{\mathrm{v}}(\vec{k})$ in each valley $\left|\Phi_{n, \tau}^{ \pm}(\vec{q})\right\rangle$ to calculate the Chern numbers

$$
\mathcal{C}_{L}^{ \pm}=\frac{1}{2 \pi} \int d^{2} q \sum_{n, \tau} F_{n, \tau}^{ \pm}(q),
$$

where $F_{n, \tau}^{ \pm}(q)=-2 \operatorname{Im}\left[\left\langle\partial_{q_{x}} \Phi_{n, \tau}^{ \pm}(\vec{q}) \mid \partial_{q_{y}} \Phi_{n, \tau}^{ \pm}(\vec{q})\right\rangle\right]$. Figure 3(b) shows the integrands of Eq. (4). Since they have azimuthal symmetry, the calculations of $\mathcal{C}_{L}^{ \pm}$involve numerical integrations of one-dimensional radial functions only. Our results for the insulating phases of the $2 \mathrm{H}-\mathrm{MoS}_{2}$ bilayer and single layer are $\mathcal{C}_{L}=2$ and $\mathcal{C}_{L}=1$, respectively, supporting the idea that the relatively weak interlayer hopping in the bilayer makes it behave approximately as a mere superposition of its two constituent monolayers, which are rotated by $\pi$ with respect to each other.

The existence of a nontrivial orbital Chern number should lead to the appearance of edge states when the
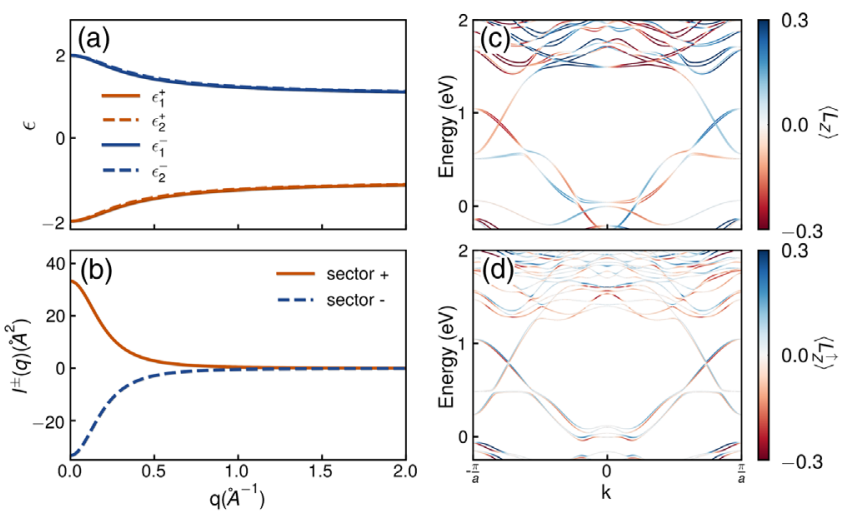

FIG. 3. (a) Positive (orange) and negative (blue) eigenvalues $\epsilon$ of the matrix $\mathbb{L}^{\mathrm{v}}(\vec{k})$ calculated as functions of the wave vector amplitude $q$ relative to valleys. (b) Integrands of Eq. (4) $I^{ \pm}(q)=$ $\sum_{n, \tau} F_{n, \tau}^{ \pm}(q)$ calculated as functions of $q$. Band structures of zigzag nanoribbons with $14.8 \AA$ in breadth calculated with the PAOFLOW Hamiltonian for a monolayer (c) and for the spin-up sector of a bilayer (d) of $2 \mathrm{H}-\mathrm{MoS}_{2}$. The color code indicates the orbital angular momentum expectation value $\left\langle L_{z}\right\rangle$. For better visualization, in the bilayer case, only the spin-up bands are shown. The spin-down band structures are shown in the Supplemental Material [46].

bulk material is cut to form a ribbon. It is well known that zigzag TMD ribbons present crossing edge states with interesting orbital properties, even though $\mathbb{Z}_{2}=0$. [65]. Figures 3(c) and 3(d) show the energy band spectra of $2 \mathrm{H}-\mathrm{MoS}_{2}$ zigzag nanoribbons, calculated with the use of PAOFLOW Hamiltonian for a monolayer and a bilayer including the orbital angular momentum expectation value $\left\langle L_{z}\right\rangle(\mathrm{k})$ for each eigenstate [66]. The energy band spectrum for a monolayer ribbon depicted in Fig. 3(c) clearly shows two pairs of orbitally polarized intravalley edge states [67] -one for each spin sector-which is compatible with the orbital Chern number $\mathcal{C}_{L}=1$. Results for the bilayer ribbon are displayed in Fig. 3(d) where we see two pairs of intravalley edge states per spin sector-which is also compatible with $\mathcal{C}_{L}=2$. For the bilayer, the presence of inversion symmetry is translated in the existence of positive and negative $L_{z}$ edge states in both valleys. For clarity, Fig. 3(d) presents the results for spin-up while the Supplemental Material [46] presents the two components.

Experimental signatures.-Now, let us briefly discuss the experimental signatures of the OHE in TMD bilayers. Typically, to characterize the OHE in these materials, one needs the same experimental setups conceived to analyze VHE in TMD bilayers, where inversion symmetry breaking is induced by a gate voltage $[25,68]$. For the bilayer in this case, both the OHE and the VHE lead to magnetic moment accumulation at the sample's edges. To provide some insights into what should be expected in such experiments, we include a gate potential in Eq. (3) given by $H_{U}=\operatorname{diag}(U, U,-U,-U)$. 

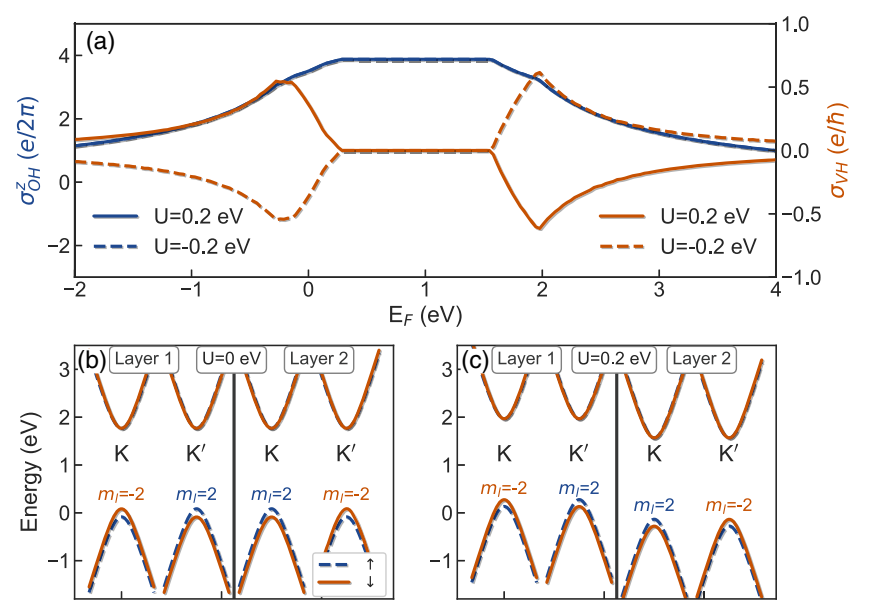

FIG. 4. (a) Orbital (blue line) and valley Hall (orange line) conductivities as a function of the Fermi energy $E_{F}$ for $U=$ $0.2 \mathrm{eV}$ (solid line) and $U=-0.2 \mathrm{eV}$ (dashed line). Schematic representation of the low energy spectrum for $U=0 \mathrm{eV}$ (b) and $U=0.2 \mathrm{eV}$ (c). The orbital polarization of the top valence bands is indicated by $m_{l}= \pm 2$ and the solid orange and dashed blue lines indicate the two spin orientations.

For a finite $U$, the inversion symmetry is broken in the bilayer and the VHE takes place. The $\mathrm{OH}$ and $\mathrm{VH}$ conductivities can be calculated using Eqs. (1)-(3). To calculate the VH conductivity (VHC), we substityte the integrand of Eq. (1) by $\Omega_{n, \vec{q}} / \hbar$, and rewrite $\sigma_{\mathrm{VH}} \equiv\left(\sigma_{\tau=+1}-\sigma_{\tau=-1}\right)$. Figure 4(a) shows our results for the $\mathrm{OHC}\left(\sigma_{\mathrm{OH}}^{z}\right)$ and $\mathrm{VHC}$ $\left(\sigma_{\mathrm{VH}}\right)$ calculated as functions of the Fermi energy $\left(E_{F}\right)$ for positive and negative values of $U$. There are clear differences between the two quantities. While the $\mathrm{OHC}$ is an even function of $E_{F}$, the $\mathrm{VHC}$ is odd. Also, the OHE is an even function of $U$, whereas the VHE is odd - the valley magnetic moment inverts when $U$ changes sign [24]. Panels (b) and (c) of Fig. 4 show the energy spectrum for $U=0$ and $U=0.2 \mathrm{eV}$, respectively. It is clear that $U$ produces a rigid energy-band shift for the two layers, without changing their orbital polarizations. The OHE should remain unchanged for small variations of $E_{F}$, but decreases when $E_{F}$ crosses any band, as individual bands in each valley contribute to the total Chern number. We have also performed DFT calculations for the OHE in the presence of an electric field applied perpendicularly to the layers [69]. The results support our low-energy analysis and are presented in the Supplemental Material [46].

Kerr rotation microscopy experiments [25] showed that a bilayer of $\mathrm{MoS}_{2}$ exhibits a sizeable Kerr rotation even in the absence of an applied gate voltage. It was argued that this unexpected behavior could originate from substrate induced inversion symmetry breaking. Recent nonlocal resistance measurements in a hexagonal boron nitride ( $h$-BN) encapsulated bilayer of $\mathrm{MoS}_{2}$ also exhibited a nonlocal signal at zero gate voltage [68]. The interpretation was the same, although one should not expect $h$-BN to cause such a large inversion symmetry breaking effect. On the other hand, the OHE could be the source of this experimental evidence and explain the unexpected signals at zero bias in the bilayers. Careful experimental analysis of Kerr rotation and nonlocal resistance measurements as functions of gate voltage may help to distinguish between the orbital and valley Hall effects in these materials. The results illustrated in Fig. 4, in light of the experiments reported in Refs. [25,68], suggest that ultrathin films of TMDs are promising platforms for exploring the OHE in 2D materials.

Final remarks and conclusion.-Our DFT calculations showed that centrosymmetric two-dimensional materials, such as a bilayer of $2 \mathrm{H}-\mathrm{MoS}_{2}$, can host an orbital Hall insulating phase in the absence of both spin and valley Hall effects. Using $\mathrm{MoS}_{2}$ as a prototype of the TMD family, we have also unveiled the topological nature of OHE in these systems and calculated the orbital Chern numbers for 2H-TMDs. Our work clarifies the interplay between orbital and valley Hall conductivity in bilayer TMDs. We found that, in the absence of a gate voltage between the layers, the magnetic moment accumulation observed in experiments should be dominated by the OHE, as VHE is zero in centrosymmetric materials. For finite bias, OHE and VHE are still decoupled and can behave as competing effects.

We acknowledge CNPq/Brazil, CAPES/Brazil, FAPERJ/Brazil, and INCT Nanocarbono for financial support. T. G. R. acknowledges funding from Fundação para a Ciência e a Tecnologia and Instituto de Telecomunicações-Grant No. UID/50008/2020 in the framework of the project Sym-Break. She thankfully acknowledges the computer resources at MareNostrum and the technical support provided by Barcelona Supercomputing Center (Grant No. FI-2020-2-0033). M. C. acknowledges the National Laboratory for Scientific Computing (LNCC/MCTI, Brazil) for providing HPC resources of the SDumont supercomputer. M. B. N. also acknowledges the High Performance Computing Center at the University of North Texas and the Texas Advanced Computing Center at the University of Texas, Austin, for computational resources. L. M. C. is supported by Project MECHANIC (Project No. PCI2018-093120) funded by the Ministerio de Ciencia, Innovación y Universidades. ICN2 is funded by the CERCA Programme/Generalitat de Catalunya and supported by the Severo Ochoa Centres of Excellence program, funded by the Spanish Research Agency (Grant No. SEV-2017-0706).Instituto Nacional de Ciência e Tecnologia em Nanomateriais de Carbono

*tarik.cysne@gmail.com

[1] B. A. Bernevig, T. L. Hughes, and S.-C. Zhang, Phys. Rev. Lett. 95, 066601 (2005). 
[2] J. Liu, Z. Ma, J. Gao, and X. Dai, Phys. Rev. X 9, 031021 (2019).

[3] D. Go, J.-P. Hanke, P. M. Buhl, F. Freimuth, G. Bihlmayer, H.-W. Lee, Y. Mokrousov, and S. Blügel, Sci. Rep. 7, 46742 (2017).

[4] T. Yoda, T. Yokoyama, and S. Murakami, Nano Lett. 18, 916 (2018).

[5] S. Beaulieu, J. Schusser, S. Dong, M. Schüler, T. Pincelli, M. Dendzik, J. Maklar, A. Neef, H. Ebert, K. Hricovini et al., Phys. Rev. Lett. 125, 216404 (2020).

[6] L. M. Canonico, T. P. Cysne, A. Molina-Sanchez, R. B. Muniz, and T. G. Rappoport, Phys. Rev. B 101, 161409(R) (2020).

[7] S. Bhowal and S. Satpathy, Phys. Rev. B 102, 035409 (2020).

[8] D. Jo, D. Go, and H.-W. Lee, Phys. Rev. B 98, 214405 (2018).

[9] J.-H. Park, C. H. Kim, H.-W. Lee, and J. H. Han, Phys. Rev. B 87, 041301(R) (2013).

[10] D. Go, D. Jo, C. Kim, and H.-W. Lee, Phys. Rev. Lett. 121, 086602 (2018).

[11] F. Xue, C. Rohmann, J. Li, V. Amin, and P. Haney, Phys. Rev. B 102, 014401 (2020).

[12] F. Castro de Lima, G. J. Ferreira, and R. H. Miwa, Nano Lett. 19, 6564 (2019).

[13] L. M. Canonico, T. P. Cysne, T. G. Rappoport, and R. B. Muniz, Phys. Rev. B 101, 075429 (2020).

[14] S. Park and B.-J. Yang, Nano Lett. 20, 7694 (2020).

[15] J. Xiao, Y. Liu, and B. Yan, arXiv:2010.01970.

[16] D. Go, F. Freimuth, J.-P. Hanke, F. Xue, O. Gomonay, K.-J. Lee, S. Blügel, P. M. Haney, H.-W. Lee, and Y. Mokrousov, Phys. Rev. Research 2, 033401 (2020).

[17] D. Go and H.-W. Lee, Phys. Rev. Research 2, 013177 (2020).

[18] Z. C. Zheng, Q. X. Guo, D. Jo, D. Go, L. H. Wang, H. C. Chen, W. Yin, X. M. Wang, G. H. Yu, W. He et al., Phys. Rev. Research 2, 013127 (2020).

[19] J. Kim, D. Go, H. Tsai, D. Jo, K. Kondou, H.-W. Lee, and Y. Otani, Phys. Rev. B 103, L020407 (2021).

[20] Y. Chen, W. Ruan, M. Wu, S. Tang, H. Ryu, H.-Z. Tsai, R. Lee, S. Kahn, F. Liou, C. Jia et al., Nat. Phys. 16, 218 (2020).

[21] F. Xue, V. Amin, and P. M. Haney, Phys. Rev. B 102, 161103(R) (2020).

[22] K. F. Mak, D. Xiao, and J. Shan, Nat. Photonics 12, 451 (2018).

[23] H. Zeng, J. Dai, W. Yao, D. Xiao, and X. Cui, Nat. Nanotechnol. 7, 490 (2012).

[24] S. Wu, J. S. Ross, G.-B. Liu, G. Aivazian, A. Jones, Z. Fei, W. Zhu, D. Xiao, W. Yao, D. Cobden et al., Nat. Phys. 9, 149 (2013).

[25] J. Lee, K. F. Mak, and J. Shan, Nat. Nanotechnol. 11, 421 (2016).

[26] Z. Gong, G.-B. Liu, H. Yu, D. Xiao, X. Cui, X. Xu, and W. Yao, Nat. Commun. 4, 2053 (2013).

[27] A. Kormányos, V. Zólyomi, V. I. Fal'ko, and G. Burkard, Phys. Rev. B 98, 035408 (2018).

[28] A. Kormányos, G. Burkard, M. Gmitra, J. Fabian, V. Zólyomi, N. D. Drummond, and V. Fal'ko, 2D Mater. 2, 022001 (2015).
[29] L. Du, M. Liao, G.-B. Liu, Q. Wang, R. Yang, D. Shi, Y. Yao, and G. Zhang, Phys. Rev. B 99, 195415 (2019).

[30] P. Hohenberg and W. Kohn, Phys. Rev. 136, B864 (1964).

[31] W. Kohn and L. J. Sham, Phys. Rev. 140, A1133 (1965).

[32] P. Giannozzi, O. Andreussi, T. Brumme, O. Bunau, M. Buongiorno Nardelli, M. Calandra, R. Car, C. Cavazzoni, D. Ceresoli, M. Cococcioni et al., J. Phys. Condens. Matter 29, 465901 (2017).

[33] J. P. Perdew, K. Burke, and M. Ernzerhof, Phys. Rev. Lett. 77, 3865 (1996).

[34] G. Kresse and D. Joubert, Phys. Rev. B 59, 1758 (1999).

[35] S. Grimme, J. Comput. Chem. 27, 1787 (2006).

[36] L. A. Agapito, A. Ferretti, A. Calzolari, S. Curtarolo, and M. Buongiorno Nardelli, Phys. Rev. B 88, 165127 (2013).

[37] L. A. Agapito, M. Fornari, D. Ceresoli, A. Ferretti, S. Curtarolo, and M. B. Nardelli, Phys. Rev. B 93, 125137 (2016).

[38] H. Kontani, T. Tanaka, D. S. Hirashima, K. Yamada, and J. Inoue, Phys. Rev. Lett. 100, 096601 (2008).

[39] I. V. Tokatly, Phys. Rev. B 82, 161404(R) (2010).

[40] V. T. Phong, Z. Addison, S. Ahn, H. Min, R. Agarwal, and E. J. Mele, Phys. Rev. Lett. 123, 236403 (2019).

[41] M. Buongiorno Nardelli, F. T. Cerasoli, M. Costa, S. Curtarolo, R. D. Gennaro, M. Fornari, L. Liyanage, A. R. Supka, and H. Wang, Comput. Mater. Sci. 143, 462 (2018).

[42] M. Costa, G. R. Schleder, M. Buongiorno Nardelli, C. Lewenkopf, and A. Fazzio, Nano Lett. 19, 8941 (2019).

[43] M. Costa, G. R. Schleder, C. M. Acosta, A. Padilha, F. Cerasoli, M. B. Nardelli, and A. Fazzio, arXiv:2006.07270.

[44] M. Costa, N. M. R. Peres, J. Fernández-Rossier, and A. T. Costa, Phys. Rev. B 102, 014450 (2020).

[45] A. M. García, E. d. Corro, M. Kalbac, and O. Frank, Phys. Chem. Chem. Phys. 19, 13333 (2017).

[46] See Supplemental Material at http://link.aps.org/ supplemental/10.1103/PhysRevLett.126.056601 which includes Refs. [47-53] for additional analyses, and details on the numerical simulations.

[47] D. Moldovan, M. Anđelković, and F. Peeters, Pybinding version 0.9.5: A Python package for tight-binding calculations (2020), https://docs.pybinding.site/en/stable/.

[48] S. M. João, M. Anđelković, L. Covaci, T. G. Rappoport, J. M. V. P. Lopes, and A. Ferreira, R. Soc. Open Sci. 7, 191809 (2020).

[49] E. Ridolfi, L. R. F. Lima, E. R. Mucciolo, and C. H. Lewenkopf, Phys. Rev. B 95, 035430 (2017).

[50] I. Souza, J. Íñiguez, and D. Vanderbilt, Phys. Rev. Lett. 89, 117602 (2002).

[51] P. Umari and A. Pasquarello, Phys. Rev. Lett. 89, 157602 (2002).

[52] A. Ramasubramaniam, D. Naveh, and E. Towe, Phys. Rev. B 84, 205325 (2011).

[53] D. N. Sheng, Z. Y. Weng, L. Sheng, and F. D. M. Haldane, Phys. Rev. Lett. 97, 036808 (2006).

[54] D. Xiao, G.-B. Liu, W. Feng, X. Xu, and W. Yao, Phys. Rev. Lett. 108, 196802 (2012).

[55] G.-B. Liu, W.-Y. Shan, Y. Yao, W. Yao, and D. Xiao, Phys. Rev. B 88, 085433 (2013). 
[56] N. Zibouche, P. Philipsen, A. Kuc, and T. Heine, Phys. Rev. B 90, 125440 (2014).

[57] J.-S. You, S. Fang, S.-Y. Xu, E. Kaxiras, and T. Low, Phys. Rev. B 98, 121109(R) (2018).

[58] D. Xiao, M.-C. Chang, and Q. Niu, Rev. Mod. Phys. 82, 1959 (2010).

[59] X. Xu, W. Yao, D. Xiao, and T. F. Heinz, Nat. Phys. 10, 343 (2014).

[60] C. L. Kane and E. J. Mele, Phys. Rev. Lett. 95, 226801 (2005).

[61] M. Ezawa, Phys. Rev. B 88, 161406(R) (2013).

[62] E. Prodan, Phys. Rev. B 80, 125327 (2009).

[63] Y. Yang, Z. Xu, L. Sheng, B. Wang, D. Y. Xing, and D. N. Sheng, Phys. Rev. Lett. 107, 066602 (2011).
[64] H. Li, L. Sheng, D. N. Sheng, and D. Y. Xing, Phys. Rev. B 82, 165104 (2010).

[65] H. Rostami, R. Asgari, and F. Guinea, J. Phys. Condens. Matter 28, 495001 (2016).

[66] D. Go, D. Jo, T. Gao, K. Ando, S. Blügel, H.-W. Lee, and Y. Mokrousov, arXiv:2011.08601.

[67] F. Zhang, A. H. MacDonald, and E. J. Mele, Proc. Natl. Acad. Sci. U.S.A. 110, 10546 (2013).

[68] Z. Wu, B. T. Zhou, X. Cai, P. Cheung, G.-B. Liu, M. Huang, J. Lin, T. Han, L. An, Y. Wang et al., Nat. Commun. 10, 611 (2019).

[69] T. Brumme, M. Calandra, and F. Mauri, Phys. Rev. B 91, 155436 (2015). 